\title{
NOTE PRÉLIMINAIRE
}

\section{INTERFÉCONDATION ENTRE TELADORSAGIA TRIFURCATA ET T. CIRCUMCINCTA}

\author{
J. CABARET*, G. MORALES** et G. LUFFAU***
}

La mise en évidence de l'existence d'une interfécondation entre ces deux Teladorsagia pose des problèmes techniques importants. Il faut disposer d'une souche pure de $T$. circumcincta et pouvoir introduire au niveau de la caillette des individus mâles de $T$. trifurcata vivants qui s'implanteront.

Une brebis fistulée de la caillette a été d'abord infestée par voie orale (212 larves de $T$. circumcincta). 10 jours plus tard, 15 mâles de $T$. trifurcata ont été introduits dans la caillette par l'orifice de la fistule de la caillette. Des coprocultures ont été réalisées sur les fèces de cette brebis; les larves du $3^{3}$ âge obtenues ont été inoculées par voie orale à un agneau. Celui-ci a été abattu et sa caillette examinée 27 jours après la date de son infestation. Trois pour cent des mâles récoltés appartiennent à $T$. trifurcata.

Seule l'existence d'une interfécondation des deux Teladorsagia (mâle de $T$. trifurcata $\mathrm{X}$ femelle $T$. circumcincta) chez le premier animal permet d'expliquer la présence de $T$. trifurcata chez le second animal.

* Institut National de la Recherche Agronomique, Station de Pathologie aviaire et de Parasitologie, Unité d'Écologie Parasitaire, Centre de Recherches de Tours, F 37380 Monnaie.

** La boratoire de Zoologie-Vers, Muséum National d'Histoire Naturelle, 61, rue de Buffon, F 75231 Paris Cedex 05 .

*** Institut National de la Recherche Agronomique, Station de Virologie-Immunologie, F 78850 Thiverval Grignon.

Accepté le 22 novembre 1983. 